\title{
NOUS, BERGSONIENS MANIFESTE DE KYOTO
}

\author{
Elie During \\ Université Paris Ouest Nanterre
}

Paul-Antoine Miquel

Université Toulouse 2

Résumé: Ce manifeste, écrit à quatre mains dans le sillage d'un colloque bergsonien organisé au Japon, vise d'abord à répondre à une question simple: que signifie, aujourd'hui, travailler à la manière de Bergson? Non pas simplement sur le corpus bergsonien, ou sur certains sujets dont sa pensée a permis de renouveler la compréhension, mais selon la méthode qu'il recommandait lui-même. Cette méthode, Bergson l'a appliquée au cours de sa recherche au prix d'efforts considérables, et singulièrement dans son rapport aux sciences positives, qu'il s'agisse des sciences du vivant ou de l'univers matériel au sens large. En se penchant notamment sur la fonction de l'imagination métaphysique, ce manifeste redessine dans les pas de Bergson les voies d'un empirisme non spéculatif et véritablement contemporain de l'intelligence scientifique.

Mots-clés: Bergson; Science; Émergence; Espace-temps; Intuition; Méthode philosophique.

Resumo: Este manifesto, escrito a quatro mãos no contexto de um colóquio sobre Bergson organizado no Japão, visa inicialmente responder a uma simples questão: que significa, hoje, trabalhar à maneira de Bergson? Não se trata de uma pergunta sobre a dedicação para com o corpus bergsoniano ou quanto a certos temas que o seu pensamento permite renovar a compreensão, mas sim sobre o método que ele próprio recomenda. Este método foi aplicado diligentemente por Bergson no curso de suas investigações, particularmente quanto ao seu trato com as ciências positivas, tanto as da vida quanto às do universo material em sentido amplo. No que diz respeito à função da imaginação metafísica, este manifesto refaz os passos de Bergson quanto a um empirismo não especulativo e verdadeiramente contemporâneo à inteligência científica.

Palavras-chave: Bergson; Ciência; Emergência; Espaço-tempo; Intuição; Método filosófico.

\section{'Nous, bergsoniens...'}

Qui sont les philosophes qui peuvent prendre aujourd'hui cette formule à leur compte? Pas nécessairement ceux qu'on a l'habitude de croiser 
dans les colloques consacrés aux “études bergsoniennes'. Quand Aristote, dans un célèbre passage de la Métaphysique consacré à la doctrine des Idées, rendait hommage à son maitre, c'était pour pousser l'intuition platonicienne dans des directions nouvelles. Il en est sorti, comme on sait, une autre philosophie. Il y aurait de quoi s'étonner si la pensée de la durée, du changement, de l'émergence du nouveau, ne finissait pas elle aussi par engendrer une philosophie pour notre temps, une philosophie qui soit autre chose qu'un retour à Bergson, un néo-bergsonisme.

On peut être bergsonien malgré Bergson, et jusqu'à un certain point contre lui. Mais la philosophie ne se décrète pas; il faut la faire. Et si le bergsonisme se distingue par sa méthode, il convient de s'assurer de sa valeur d'usage. Il faut voir ce qu'elle peut donner aujourd'hui, en fonction des problèmes qui sont les nôtres. Qu’à cet égard les meilleurs spécialistes de Bergson ne soient pas toujours eux-mêmes très bergsoniens, c'est ce qui apparaît dès qu'on s'intéresse à la manière dont ils travaillent en fait. Gilson a bien résumé le problème : "Les vrais bergsoniens ne sont pas ceux qui répètent les conclusions, ce sont plutôt ceux qui, à son exemple, refont à leur propre compte, et sur des terrains différents, quelque chose d'analogue à ce qu'il a fait ${ }^{1}$.'

Nous, bergsoniens, nous avons lu et relu Bergson; nous avons suivi les méandres de sa réception; nous l'avons défendu contre ses détracteurs ; nous avons corrigé des malentendus, restauré le relief de son œuvre, éprouvé la singularité et le tranchant de ses thèses, la subtilité de sa manière de penser, exigeante et "difficultueuse' sous la fausse apparence du relâchement (“Comment quelques-uns ont-ils pu s'y tromper?’)... Tout cela était nécessaire. Mais l'heure n'est plus de redonner une seconde jeunesse à la 'philosophie nouvelle'. Bergson est bien là parmi nous: il a eu son moment, il ne manque pas d'amis. La question est de savoir comment poursuivre et prolonger l'effort d'une pensée, quitte à en renouveler le programme.

Bergson a été interprété de diverses manières: il s'agit maintenant de le transformer. Et pour commencer, de le mettre au travail dans un contexte qui n'est évidemment plus celui de son siècle.

1 Étienne Gilson, 'Souvenir de Bergson', Revue de métaphysique et de morale, 64, 1959, p.136. En écrivant cela, Gilson avait à l'esprit un exemple précis : 'Le plus apparemment "bergsonien" de ceux qui portèrent la marque de son influence, Édouard Le Roy, s'est toujours défendu d'enseigner la même doctrine que celle du philosophe auquel il a rendu tant et de si fervents hommages. [...] Ce n'était d'ailleurs pas le trahir, c'était plutôt l'imiter, car chaque fois qu'il abordait un nouveau problème, Bergson lui-même ne s'en approchait pas sans lui consacrer un effort nouveau, après une enquête nouvelle.' Sur la portée du nom 'bergsonien', voir Giuseppe Bianco, Après Bergson: portrait de groupe avec philosophe, Paris, Presses universitaires de France, coll. Philosophie française contemporaine, 2015. 
C'est pourquoi nous plaidons pour un bergsonisme élargi.

\section{Pour un bergsonisme élargi}

En quel sens? D'abord et avant tout, dans la direction des sciences et des savoirs positifs de notre temps. La méthode bergsonienne risque à son tour de n'être qu'une dialectique de concepts si on la cantonne à ses applications intra-philosophiques (critique des faux problèmes, reformulation “en durée' des grandes questions de la métaphysique: déterminisme et contingence, esprit et matière, problème du monisme, etc.), en la coupant du rapport effectif à la production scientifique qui a été son aiguillon constant. La métaphysique ne se construit pas dans le vide, elle n'est jamais a priori. L'intuition est réflexion, rappelle Bergson. Mais réflexion sur quoi? Invoquer ici l'expérience vécue n'est pas d'un grand secours si l'on n'y inclut pas aussitôt la riche contexture des trames d'expérience élaborées par l'intelligence technique et scientifique, inséparables d'une mise en forme symbolique du donné. Bergson a voulu l'expérience intégrale. Il serait curieux d'en exclure par principe l'essentiel de notre activité pensante.

Cette nécessité de réengager le bergsonisme dans la voie d'une confrontation aux sciences vivantes -aux sciences en train de se faire- illustre une exigence plus générale. Ce dont nous avons besoin aujourd'hui, ce n'est pas un nouveau commentaire, mais un programme de recherche ajusté aux problèmes du moment, en résonance par rapport à une constellation de questions bergsoniennes, mais aussi, le cas échéant, en écart par rapport à certains choix méthodologiques. Pour mener à bien un tel projet à partir de Bergson, il n'est pas nécessaire, il est même préférable parfois, de ne pas parler sa langue. Répétons-le, il ne s'agit pas tant de lire ou de relire Bergson; il s'agit surtout de le traduire. Traduire Bergson, c'est-à-dire d'abord desserrer l'étau de ses mots, plonger ses énoncés dans de nouveaux milieux, les projeter sur de nouvelles trames. Ce travail de déformation réglée passe aussi par la diversité des vocables et des traditions philosophiques. Oui, il faut continuer à confronter Bergson avec la phénoménologie et la philosophie analytique, méthode contre méthode. Il faut imaginer, pourquoi pas, un Bergson analytique, un Bergson philosophiquement glabre, comme dirait Deleuze: non pour le plaisir pervers de le faire se retourner dans sa tombe, mais pour éprouver la spécificité d'un positionnement et d'une méthode, pour mettre ensuite en perspective, selon un angle propre, certains champs de recherche, en 
définissant pour chacun de ces champs, dans chaque situation critique, un mode d'articulation approprié entre philosophie et disciplines positives ${ }^{2}$.

Promouvoir un bergsonisme élargi, c'est se libérer de la mauvaise conscience des continuateurs qui craignent si fort d'être des disciples. C'est être bergsonien autrement, sans avoir à se faire le gardien d'une orthodoxie. Rien ne nous interdit de recomposer la doctrine, de recombiner entre eux les concepts bergsoniens pour pousser plus loin une intuition ou lui trouver un autre angle d'incidence. Rien ne nous interdit non plus d'enrichir ou de transformer certaines analyses au contact d'autres programmes philosophiques, pour autant qu'ils croisent la pensée bergsonienne sur des problèmes communs. Le bergsonisme élargi sera donc un bergsonisme réfracté. Et le risque, bien entendu, est qu'à un certain moment plus rien ne passe, qu'on ne tienne plus qu'un effet de mirage, une espèce de bergsonisme virtuel ${ }^{3}$. Mais c'est peut-être là le prix de toute expérimentation réelle: 'la force de toute pensée forte, écrivait Rancière à propos de Deleuze, est aussi sa capacité de disposer elle-même son aporie, le point où elle ne passe plus ${ }^{4}$.

Les paragraphes qui suivent dessinent les grandes lignes et les enjeux de ce bergsonisme élargi, tout en indiquant quelques-uns des lieux critiques sur lesquels devra se concentrer la recherche.

\section{Un empirisme non spéculatif: suivre les lignes de faits}

Insistons pour commencer sur un point capital. Bergson se réclamait comme on sait d'un 'empirisme vrai'. Il parlait d'une "métaphysique positive'. L'expérience philosophique se distingue de la spéculation métaphysique -et d'un certain rapport immédiat au tout de l'expérience dont cette dernière pourrait se réclamer-, en ceci qu'il n'y a pas de philosophie qui ne passe par des trames symboliques constituées au contact de faits et de savoirs positifs.

Les 'données immédiates' l'ont parfois fait oublier: il n'y a pas d'expérience philosophique originaire, phénoménologiquement pure; ou du moins, pas d'originaire qui ne s'éprouve d'emblée à travers des faits déjà partiellement constitués en objectivités par l'œuvre de l'intelligence. On ne comprendrait pas, autrement, que Bergson se soit mis en tête de se forger dès le

2 Le colloque organisé au Japon autour de Matière et Mémoire par Yasushi Hirai, Hisashi Fujita et Shin Abiko fut, à cet égard, une expérimentation exemplaire ('The Anatomy of Matter and Memory: Bergson and Contemporary Theories of Perception, Mind and Time', 7th International Workshop of the Project Bergson in Japan, Tokyo et Kyoto, 10-13 décembre 2015). Ce manifeste ébauché sur place, entre deux sessions, en est le produit direct.

${ }^{3}$ Le premier chapitre de Matière et Mémoire décrit précisément ce phénomène de 'réflexion totale' qui sert d'analogie pour la perception pure (Matière et Mémoire, Paris Presses universitaires de France, 2007, p.34).

${ }^{4}$ Jacques Rancière La Chair des mots, Paris, Galilée, 1999, p.203. 
départ des instruments critiques, en cherchant à démêler l'espace et la durée. La durée pure est le terme d'un effort; nous commençons toujours au milieu, dans le mélange. Le 'tournant de l'expérience' est un point de fuite; il n'offre pas un premier principe. La grammaire de l'expérience philosophique ne saurait donc être a priori. Elle ne prend pas non plus la forme classique d'un transcendantal.

Cela ne veut pas dire pour autant qu'il n'y ait pas de grammaire, et que tout se réduise chez Bergson à des opérations critiques. Cette grammaire, il faut parfois la chercher en filigrane dans les textes. C'est une grammaire constructive plutôt que donnée, ou déduite. Il convient de la comprendre dans sa relation à une imagination métaphysique qui fournit, rétrospectivement, quelque chose comme un dessin: une vision élaborée en schème de réalité. La précision métaphysique passe par le flou de cette vision et par l'indécis de ce schème. Elle ne pense au propre que lorsqu'elle parle au figuré5. C'est ce qui fait toute la difficulté de la langue de Bergson, toute l'ambiguité de sa prose 'imagée'. Une image 'n'est pas un ornement, c'est une suggestion de représentations qui, pour être fécondes, doivent suggérer des recherches ${ }^{6}$. '. Et c'est pourquoi il en faut toujours plusieurs: une variété d'images, si possible disparates, dont la disparité même permettra de corriger ce que chacune pourrait avoir d'excessif ou d'unilatéral, tout en maintenant l'attention sur l'essentiel. Car l'image est d'abord un point de visée, et elle remplit à cet égard exactement le même rôle que les lignes de faits. Celles-ci trouvent en effet leur pleine signification philosophique lorsqu'elles viennent à se recouper.

C'est ainsi que des phénomènes à première vue aussi disparates que la paramnésie (sentiment de 'déjà vu'), la 'vision panoramique' des mourants, ou encore l'insistance obtuse du "mot sur le bout de la langue', se mettent à fonctionner comme autant d'indices du mode d'existence d'un passé immémorial qui se produit et se conserve en soi à mesure que la réalité se fait. Les faits ne sont pas seulement 'têtus', selon le mot de Lénine: ils sont surtout disjoints et éparpillés, de sorte qu'il faut commencer par les rassembler, les arranger selon des 'lignes de faits', pour tâcher ensuite, au prix de tâtonnements parfois laborieux, et sans annuler l'hétérogénéité du donné, de les faire converger vers un même point de visée. Si elle n'était pas soutenue par ce patient travail de mise au point optique, la conservation intégrale du passé resterait une image obscure. Il s'agit dans tous les cas de faire le tour de la question en occupant tour à tour plusieurs positions stratégiques: 'l'intuition

5 Voir l'introduction au dossier 'Bergson et la science', P.-A. Miquel et F. Worms (dir.), in Annales Bergsoniennes, n.3, Paris, Presses universitaires de France, coll. Épiméthée, 2006.

6 Entretien de 1938 avec Bergson, cité par Lydie Adolphe, La Dialectique des images, Paris, Presses universitaires de France, 1951, p.4. 
n'est pas une inspiration venue d'en haut; c'est une démarche qui consiste à sauter au centre, après avoir visé de tous les points ${ }^{7}$.

\section{Engagement ontologique et distance critique: le rapport aux sciences}

Si la pensée de Bergson ne s'est jamais transformée en néobergsonisme, une inspiration bergsonienne traverse pourtant toute la philosophie française contemporaine, de Canguilhem à Deleuze en passant par Simondon, Ruyer, Merleau-Ponty et Foucault, pour ne citer qu'eux. Elle se marque à l'importance donnée aux lignes de faits, ainsi qu'aux sciences positives qui permettent d'en appréhender le détail.

Il faut à cet égard rappeler deux points hélas constamment négligés dans la réception du bergsonisme.

$1^{\circ}$ Contrairement aux idées reçues, la connaissance scientifique, pour Bergson, n'est pas nécessairement artificielle et symbolique. Elle ne serait pas si efficace si elle ne portait déjà, par elle-même, un engagement ontologique: dans son ordre, 'la science touche à l'absolu'. Même les mathématiques, à certains égards, 'ne sont point du tout un jeu, mais une véritable prise de contact avec l'absolu ${ }^{8}$. 'Je voudrais savoir s'il existe, parmi les conceptions modernes de la science, une théorie qui mette plus haut la science positive".

$2^{\circ}$ L'intuition n'est pas l'instrument de précision qu'on a parfois décrit, cette espèce de rayon laser par lequel la pensée se rendrait capable d'éprouver, de plain-pied, la réalité mouvante du devenir. L'intuition donne l'impulsion, mais elle est par elle-même vague. Elle ne trouve à se préciser qu'en se localisant, en se resserrant ${ }^{10}$. Elle a besoin, on vient de le rappeler, de suivre des lignes de faits, de passer à travers des trames symboliques, de se projeter dans des formats conceptuels ${ }^{11}$.

\footnotetext{
${ }^{7}$ Lydie Adolphe, op.cit., p.4.

8 Henri Bergson, 'Discussion à la Société française de philosophie, 28 novembre 1907, in Écrits, Paris, Presses universitaires de France, 2011, p.353.

9 lbid.

10 Le 'resserrement' des problèmes est complémentaire du procédé du recoupage. Le meilleur exemple d'une telle stratégie est fourni par Matière et Mémoire, avec le choix de se concentrer sur le phénomène particulier de la mémoire, et plus spécialement sur le cas exemplaire de l'aphasie, qui permet de cerner la question du dualisme de l'âme et du corps 'au point où l'activité de la matière frôle celle de l'esprit'.

11 Camille Riquier insiste bien sur ce point dans Archéologie de Bergson, Paris, Presses universitaires de France, 2009, p.253 s.
} 
Il y a là davantage qu'une nécessité pratique. Sans doute, l'intuition ne se communique que par l'intelligence; pour se transmettre, il lui faut certes 'chevaucher sur des idées ${ }^{12}$. Mais Bergson va plus loin:

[D]ès que nous avons aperçu intuitivement le vrai, notre intelligence se redresse, se corrige, formule intellectuellement son erreur. Elle a reçu la suggestion; elle fournit le contrôle. Comme le plongeur va palper au fond des eaux l'épave que l'aviateur a signalé du haut des airs, ainsi l'intelligence immergée dans le milieu conceptuel vérifiera de point en point, par contact, analytiquement, ce qui avait fait l'objet d'une vision synthétique et supra-intellectuelle ${ }^{13}$

Et mieux encore:

L'intuition dont je parle ne peut le plus souvent entrer en jeu qu'après qu'on a étudié, approfondi, critiqué, souvent même accru tout ce que la science positive a réuni de faits sur un certain point ${ }^{14}$.

La science touche à l'absolu, et l'intuition a besoin d'elle, non seulement pour se transmettre, mais déjà pour opérer. Pour ces deux raisons, la science est bien davantage qu'un terrain d'application de la méthode bergsonienne. Elle ne se limite surtout pas à cette fonction de repoussoir que remplit trop souvent, dans le bergsonisme scolaire, la référence à l'intelligence fabricatrice, incapable de comprendre la vie, 'spatialisant' et dénaturant tout ce qu'elle touche. La science n'est pas un prétexte ou un faire-valoir pour une méthode générale. Elle en est, véritablement, la condition d'effectivité. C'est dire aussi que cette méthode ne se confond ni avec un simple accompagnement herméneutique des gestes de la pensée scientifique, ni avec une élucidation de l'ontologie implicite des théories. La philosophie, affirme Bergson, doit pouvoir 'se mouler sur la science'. Mais cette intimité est le signe d'une complémentarité réelle, sans subordination. Et cela suppose que l'intuition introduise par rapport aux constructions scientifiques une distance critique, quoique d'une tout autre manière que la phénoménologie ou la métaphysique analytique.

C'est la première caractéristique de 'l'intuition comme méthode', pour reprendre la formule de Deleuze. L'intuition se manifeste d'abord par sa puissance de refus. Elle critique les faux concepts, elle dissout les 'mauvais

${ }_{12}$ Cf. L'Évolution créatrice, Paris, PUF, 2007, p.239: 'la dialectique est nécessaire pour mettre l'intuition à l'épreuve, nécessaire aussi pour que l'intuition se réfracte en concepts et se propage à d'autres hommes'.

${ }^{13}$ Henri Bergson, La Pensée et le mouvant, Paris, Presses universitaires de France, 2007, p.67.

14 Note du 16 mai 1912 à propos du livre de Joseph Deseymard, La Pensée d'Henri Bergson, paru en novembre 1912 (Fonds Doucet, BGN 2966). Cité par C. Riquier, "Voir et cependant ne pas croire" Intuition et méthode chez Bergson', Transparaître N 1, 'l'intuition', décembre 2007, p.192. 
mixtes': c'est le moment analytique, essentiel chez Bergson. Elle fait la part du grain scientifique et de l'ivraie scientiste. Elle redresse au besoin de fausses analogies ou des généralisations hâtives pour mettre à nu l'inconsistance d'une certaine métaphysique des savants, le plus souvent inconsciente. Peut-être même peut-elle prétendre réformer certains procédés, certaines habitudes de la science au nom même des faits qu'elle découvre et des questions qu'elle suscite. L'engagement ontologique des sciences a pour prix un certain interventionnisme philosophique: un bergsonisme qui ne contribuerait en rien à l'amélioration de la science ne vaudrait pas une heure de peine ${ }^{15}$. Mais en aucun cas l'intuition n'a pour tâche de fournir à la science des 'fondements' métaphysiques dont celle-ci se passe fort bien. Et elle n'a pas davantage vocation à tirer de la science des vues cosmologiques ou une conception du monde, selon un mouvement qui reviendrait à prolonger le discours scientifique par un effort de synthèse mené dans la même direction que lui, mais à un niveau de généralité encore plus grand. Ce serait condamner le philosophe à toujours retarder sur les sciences, dont il ne peut saisir qu'un état momentané.

Bergson a donc tenté un nouage inédit entre métaphysique et science. Il l'a fait du point de vue de questions philosophiques qui ont leur autonomie, et c'est ce qu'il y a de plus difficile à faire entendre aux savants, généralement aussi prompts à se forger leur 'philosophie' qu'à la répudier comme inutile. Mais ces questions, la science contribue dans tous les cas à les préciser en réclamant des différenciations fines.

Je vois dans la métaphysique à venir, une science empirique à sa manière, progressive, astreinte comme les autres sciences positives, à ne donner que pour provisoirement définitifs, les derniers résultats où elle aura été conduite par une étude attentive du réel ${ }^{16}$.

Ceci étant posé, dans quelles directions fera-t-on porter l'effort? Quelles sont les questions qui nous occupent aujourd'hui? Quelles lignes de faits s'offrent à la réflexion philosophique?

\footnotetext{
${ }^{15}$ Bergson n'envisage évidemment pas que le philosophe puisse contribuer directement à la formulation d'une théorie scientifique. Mais il ne cantonne pas pour autant son activité à un simple exercice de clarification épistémologique. Le philosophe peut proposer dans certains cas un arbitrage entre théories concurrentes, eu égard à leur plus ou moins grande capacité à rendre compte de l'expérience intégrale: c'est ainsi que Bergson est 'interventionniste' sur le terrain de la biologie, pour reprendre une expression de Jean Gayon. En suivant les lignes de faits, il arrive aussi que le philosophe en révèle de nouveaux. On a cité plus haut ce passage étonnant: l'intuition n'entre en jeu qu'une fois 'qu'on a étudié, approfondi, critiqué, souvent même accru tout ce que la science positive a réuni de faits sur un certain point'.

${ }^{16}$ Henri Bergson, 'Le parallélisme psycho-physique et la métaphysique positive', in Écrits philosophiques, Paris, Presses universitaires de France, 2011, p.249.
} 
Où sera-t-on conduit? Nul ne le sait. Nul ne dira même quelle est la science dont relèveront les nouveaux problèmes. Ce pourra être une science à laquelle on est totalement étranger ${ }^{17}$.

\section{$\mathrm{Ni}$ réductionnisme, ni émergentisme}

Prenons l'exemple du vivant, ou de l'organisation. On a redécouvert le sens du vitalisme bergsonien, parfois obscurci par une fixation exclusive sur l'image de l'élan, ou par le caractère un peu systématique de la manœuvre dialectique consistant à renvoyer dos-à-dos les partisans du mécanisme et ceux du finalisme. D'un point de vue critique, le point à retenir est que l'approche bergsonienne donne des clés pour sortir de l'alternative entre une vision physicaliste réductionniste de la nature et une vision émergentiste et hiérarchisée (pour la nature elle-même, comme pour les relations entre nature et culture). L'émergentisme peut se développer tantôt en termes de niveaux de réalité, tantôt en termes de niveaux d'explication (des propriétés supérieures 'survenant' sur des propriétés plus fondamentales). Mais du point de vue où se place Bergson, cela ne change rien à l'affaire. Car l'être ne saurait se ramener à un plan de base : il y a d'emblée une pluralité - sans doute irréductible - de modes d'existence, et cette pluralité ne se distribue pas en couches correspondant à des niveaux de complexité croissants, couronnés par la 'région conscience'; elle n'est pas stratifiée de manière simple. Comme le dit Ruyer, 'le "nouveau" se forme à chaque instant et partout, mais il n'y a pas de couches superposées [...], apportant chacune un "novum" caractéristique ${ }^{18}$ ', (Il va sans dire que le bergsonisme nous préserve également d'une autre forme de réductionnisme, ou de régionalisme: celui propre à la philosophie de la conscience husserlienne, ou encore à l'ontologie phénoménologique du Dasein.).

En somme, l'approche bergsonienne du problème du vivant part de l'idée que nous sommes toujours déjà plongés dans des niveaux enchevêtrés de durée, non pas comme disait Deleuze dans un 'plan d'immanence', ou même dans une pluralité de tels plans, mais plutôt dans des redoublements d'immanence. Nous le sommes d'abord, comme l'a noté Frédéric Worms, parce qu'il y a deux sens de la vie ${ }^{19}$ et que le point de départ de l'Évolution créatrice consiste à opérer ce que l'on pourrait appeler un tournant cosmologique dans la pensée bergsonienne. Ce tournant signifie qu'il n'est plus possible de faire l'expérience de l'immanence

\footnotetext{
${ }^{17}$ Henri Bergson, La Pensée et le mouvant, op. cit., p.72.

${ }^{18}$ Raymond Ruyer, Néo-finalisme, rééd. Paris, PUF, 2012, p.272-273.

${ }^{19}$ Frédéric Worms, Bergson ou les deux sens de la vie, Paris, Presses universitaires de France, 2004. Cette thématique résonne encore dans un livre informé par les recherches de Winnicott et de Bowlby sur les formes concrètes du lien et de l'attachement : La Vie qui unit et qui sépare, Paris, Payot, 2013.
} 
de la durée à nos vécus de conscience sans faire en même temps l'expérience de 'la durée immanente au tout de l'univers', avec laquelle la conscience sympathise. L'exemple paradigmatique n'est autre que 'le morceau de sucre', lequel n'est pas une fois, mais deux fois de l'absolu ${ }^{20}$.

Ces redoublements permettent à une durée de se constituer par enveloppement ou contraction de durées plus ou moins intenses (c'est le thème à résonance phénoménologique de la situation), mais simultanément et à des degrés divers, par extraposition ou projection dans des durées qui l'enveloppent elle-même (c'est le thème, moins apparent mais bien présent, de la perspective, sur lequel on reviendra plus loin). Bergson introduit des distinctions fondamentales qui permettent de se repérer dans ce paysage d'enchevêtrements où règne la causalité réciproque: sur le plan statique, c'est la distinction entre le clos et l'ouvert; sur le plan dynamique, c'est la distinction entre répétition itérative et répétition créatrice.

Or nous pensons aujourd'hui que les niveaux enchevêtrés de durée sont une caractéristique fondamentale, un des paramètres d'ordre susceptibles de spécifier l'organisation biologique. Le concept d'immanence redoublée prend une forme encore plus explicite chez Simondon avec l'exigence qu'il formule de concevoir l'individuation biologique comme un 'théâtre' dans lequel elle est en même temps acteur. Et nous retrouvons cette même logique de pensée chez Bailly, Longo et Montévil, lorsqu'ils proposent de comprendre la transition du physique au biologique à travers l'idée qu'un organisme vivant se développe, se répare et se reproduit dans un espace de criticité qui viendrait redoubler l'espace de ses propriétés physiques standard.

\section{Le virtuel et le possible}

Dans une perspective bergsonienne, le virtuel est réel, mais le virtuel n'est pas le simple possible. La vie, la mémoire, en tant qu'elles sont virtuelles ou ne cessent de se virtualiser, conduisent à une réévaluation de la relation modale entre le possible et l'actuel, entre ce qui est et ce qui peut être. C'est-àdire qu'il ne suffit pas d'intégrer ce qui peut être dans le champ de ce qui est de ce qui, comme tel, peut être soumis à la quantification ou au calcul-, pour rendre compte de la dimension virtuelle qui sous-tend les processus réels.

Par exemple, le fait de prendre en compte l'espace des états d'un système biologique dans un univers de possibles intégrant l'aléatoire et les probabilités ne suffit pas à expliquer la dimension de radicale nouveauté et d'imprévisibilité qui caractérise un tel système. L'évolution de la vie, tout

20 Voir la remarquable analyse qu'en donne Pierre Montebello dans L'autre métaphysique: essai sur la philosophie de la nature, Ravaisson, Tarde, Nietzsche et Bergson, Paris, Desclée de Brouwer, 2003. 
comme la mémoire humaine, crée à chaque instant de nouveaux possibles. Elle rétroagit en temps réel sur l'espace des possibles qui est censé l'expliquer. On est ainsi conduit à distinguer conceptuellement ce qui 'est autre' et ce qui 'peut être', le simplement possible et le contingent, par nature imprévisible. Ce point de grammaire, on s'en doute, n'est pas sans répercussion sur le terrain des théories de la décision, ou de la philosophie de l'action.

\section{Pour une logique élargie}

L'ontologie bergsonienne réclame - comme chez Simondon, d'une autre manière - un élargissement de la logique. Cet élargissement n'implique pas qu'on remette en cause le principe de contradiction, mais il nous oblige à rejeter le principe du tiers exclu: Non non $A$ n'est pas identique à $A$.

Cette affaire commence chez Bergson avec 'la théorie des deux ordres'. La matière n'est pas du Rien, elle est une autre forme de durée que la vie. Elle est répétition, là où la vie est invention et création, dépassement de soi, c'est-à-dire de ce qu'il y a de matériel en elle. Mais réciproquement, la matière fait partie de la vie, elle est enveloppée par elle. On rejoint par cette voie la question de l'émergence. L'organisation est-elle quelque chose d'émergent sur un substrat physique, ou bien n'est-ce pas plutôt l'inverse? Le physique ne serait-il pas mieux décrit comme le lieu de l'annulation ou du ralentissement des interactions constitutives du biologique? On tient là un bel exemple d'extension non conservatrice: pour étendre le physique au-delà de son ordre, il faut se rendre compte que cette base n'était elle-même que le produit d'une idéalisation.

Chez Canguilhem, de même, la pathologie n'est pas Rien, mais une autre allure de vie. Ou si l'on préfère, la pathologie est une physiologie déviée. Ce qui signifie qu'on ne peut pas la réduire à la physiologie. Et la vie inversement est 'polarité dynamique', ce qui ne signifie pas simplement qu'elle s'oppose à la mort, ou encore que la santé s'oppose à la pathologie, mais qu'il y a dans la santé même une dimension pathologique, que la mort est dans la vie.

Les choses sont encore plus explicites chez Simondon. 'Pour penser l'individuation, écrit-il, il faut penser l'être non pas comme substance, ou matière, ou forme, mais comme système tendu, sursaturé, au-dessus du niveau de l'unité, ne consistant pas seulement en lui-même, et ne pouvant pas être adéquatement pensé au moyen du principe du tiers exclu ${ }^{21}$. Ce qui revient à dire que le devenir n'est pas du rien, ou que l'être n'est pas le plein. En même temps qu'il y a un être du devenir, il y a un devenir de l'être. Et l'être, précise

${ }^{21}$ Gilbert Simondon, L'Individuation à la lumière des notions de forme et d'information, Grenoble, Jérôme Millon, 2005, p.25. 
Simondon, peut se déphaser par rapport à lui-même ${ }^{22}$. En refusant le tiers exclu, on est en position de comprendre que ce qui vient en premier est le virtuel, et non l'actuel. Le virtuel, c'est ce qui est autre, et l'actuel, c'est ce qui est autre que l'être-autre.

\section{Présentisme et éternalisme}

C'est sur cette base qu'il convient d'envisager l'apport critique de Bergson aux débats contemporains concernant la métaphysique du temps. Dans les quartiers analytiques notamment, lorsque les 'présentistes' et les 'éternalistes' s'opposent sur la question de savoir quelle extension donner au verbe 'exister' (le présent seul existe-t-il? ou bien le passé également, et peutêtre même le futur?), il est clair que leur désaccord repose sur un présupposé commun, largement non interrogé. On suppose en effet que le passé et le présent sont foncièrement homogènes, que le passé ne diffère du présent que parce qu'il n'est plus le présent qu'il a été, de sorte que ce passé et ce présent ont exactement le même contenu, et ne peuvent différer que d'un point de vue modal. À charge pour chacun d'expliquer ensuite cette différence, en invoquant tantôt un mystérieux 'passage' du temps (qu'on pourra évidemment préciser en analysant divers 'modèles' du flux de conscience ${ }^{23}$ ), tantôt l'effet de perspective induit par une situation en elle-même contingente (c'est le caractère indexical $\mathrm{du}$ 'maintenant'). Si en revanche le présent et le passé diffèrent en nature, tout comme l'actuel et le virtuel selon Bergson, c'est toute l'ontologie temporelle qui est à revoir. Mais le prisme des débats analytiques obligera alors à préciser les choses, pour ne pas se contenter trop vite de formules gnomiques, opaques à force d'être répétées, comme celle qui énonce que le passé se conserve intégralement 'en soi'. Il faudra se mettre en quête de nouvelles lignes de faits, en suivant si nécessaire les derniers développements des neurosciences.

\section{Réexaminer la relation entre durée et espace}

Autre exemple, autre terrain. Nous disions qu'il ne fallait pas hésiter à desserrer l'étau de la langue bergsonienne. Commençons par la durée, le concept central du bergsonisme. On l'a généralement réduit à celui d'une multiplicité qualitative ou hétérogène, dépourvue de toute dimension numérique. Cette idée a fait son temps, comme le montrent une série de

22 '[L]'être possède une unité transductive, c'est-à-dire qu'il peut se déphaser par rapport à lui-même, se déborder lui-même de part et d'autre de son centre' (ibid., p.31).

${ }^{23}$ C'est par exemple la perspective adoptée par Barry Dainton dans ses études consacrées à l'expérience temporelle. Yasushi Hirai en a donné une présentation et une lecture critique des plus intéressantes à l'occasion du colloque 'The Anatomy of Matter and Memory' de décembre 2015. 
travaux récents ${ }^{24}$. La durée n'est pas l'autre du nombre; elle est un 'nombre obscur', un 'nombre spécial', c'est-à-dire non spatial. Cette idée porte toute une arithmétique qualitative, capable de décrire les phénomènes physiques sans annuler la dimension temporelle inhérente à leur devenir. La mesure y apparaît comme une manière de se rapporter au rythme interne du changement.

Parmi les autres 'gros mots' du bergsonisme, celui de 'spatialisation' n'est pas le moins embarrassant. Qu'est-ce que l'espace? La fonction critique de cette catégorie est assez claire; il est inutile d'y revenir. Mais qu'en est-il alors de la pluralité des espaces concrets et abstraits associés aux modes de vie humains et animaux, aux constructions scientifiques ou artistiques? Y a-t-il de ce côté des lignes de faits qui puissent intéresser le philosophe? Bergson luimême s'est attaqué à une variété particulière d'espace abstrait, l'espace-temps à quatre dimensions (et à signature pseudo-euclidienne) des physiciens relativistes. Il n'y a vu, en somme, qu'une extension universelle du procédé de figuration géométrique du mouvement illustré par l'usage ordinaire des graphes ou des courbes d'évolution. Aurait-il dit la même chose des espaces de configuration, ou des espaces de phases couramment utilisées dans la physique contemporaine? Et si la matière tend à l'espace comme vers une limite idéale, comment situer, dans ce schème métaphysique de l'extensif, la diversité de fait des constructions géométriques: espaces non euclidiens, à $n$ dimensions, complexes, discrets, etc.? Quel statut donner à la théorie des ensembles? N'y a$\mathrm{t}$-il là qu'une extension naturelle de la conception bergsonienne - très moderne en son temps - des objets mathématiques comme systèmes de relations entre objets élémentaires (points, instants)? Que dire enfin des aspects purement topologiques que présente l'espace mathématique? La critique bergsonienne de la mesure, conçue à l'origine sur des exemples aussi élémentaires que ceux de l'Esthétique transcendantale, a-t-elle même prise sur ces formes plus élaborées mais effectives de spatialité?

Pour mille raisons, le traitement bergsonien de la relation entre durée et espace a de quoi nous laisser insatisfaits. Si l'on prend au sérieux l'idée selon laquelle l'intelligence ne se contente pas de fabriquer et de manipuler, mais s'inscrit véritablement dans un horizon ontologique, celui de la matière et du monde physique; si l'univers dure, comme Bergson y insiste souvent, si l'espace n'est lui-même que le degré le plus relâché de la matière, il est clair que l'espace réel - risquons ce terme, par symétrie avec le 'temps réel' - peut avoir un contenu beaucoup plus riche que celui présenté dans les Données immédiates. Rien, sinon l'habitude ancrée par l'usage répété de 'conventions commodes', selon l'expression de Poincaré, ne nous condamne à tourner éternellement

${ }^{24}$ Nous renvoyons notamment aux études de David Lapoujade, Hisashi Fujita et Sébastien Miravète. 
dans le cercle de l'imagination euclidienne. Il reste alors à voir si les déterminations multiples de la spatialité peuvent entièrement se résoudre dans le schème intensif mis en place à partir de Matière et Mémoire - celui des degrés de durée, ou des rythmes variablement contractés.

\section{Le problème de la coexistence: le local et le global}

Contrairement à la présentation qu'en donne volontiers Bergson, cette question du statut de l'espace est au cœur de sa discussion avec Einstein. Non seulement parce qu'il y est question, de façon centrale, de la simultanéité, et tout autrement que dans les premiers livres où cette notion était définie comme l'intersection du temps avec l'espace, mais parce qu'avec le 'temps réel' de Durée et Simultanéité, Bergson entend projeter la durée sur un certain aspect du temps mesuré. Oui, le temps réel - cette figure de la durée mise en avant dans Durée et Simultanéité - est bel et bien un temps mesuré. La distinction entre temps réels et temps fictifs (symboliques, purement mathématiques) passe au sein même du temps mesuré. On ne s'en est pas beaucoup avisé. Pourtant, s'il en allait autrement, Bergson n'aurait même pas pu songer à refuser les conséquences métriques du fameux 'paradoxe des jumeaux' attribué à Langevin. Or ce qu'il conteste - manifestement à tort: toutes les lignes de fait convergent sur ce point -, c'est bien l'idée que le jumeau accéléré dans son astronef puisse donner une mesure différente de la durée écoulée au cours de son voyage, durée qui est donc supposée substantiellement identique mais aussi métriquement égale à celle de son frère resté sur terre. En termes plus précis: Bergson considère qu'il n'y a qu'un seul intervalle de 'temps propre' entre les deux moments que marquent la séparation et les retrouvailles des jumeaux, et ceci précisément parce qu'il est question non pas de durées en général, mais de temps mesurés par des observateurs humains, conscients, capables de rapporter leurs mesures au flux de leur durée vécue.

Ce qui doit nous intéresser dans cette discussion, c'est le problème général. Le temps réel n'est pas nécessairement vécu: la seule chose que réclame Bergson est qu'il puisse l'être. En revanche, il est essentiel qu'il s'agisse par nature d'un temps mesuré25. Il ne suffit donc pas de dire que la durée pure, à la lettre, ne se mesure pas; il faut encore comprendre que la durée vécue peut sortir d'elle-même, adopter un poste d'observation sur le terrain même de la mesure, afin de se donner une perspective sur les autres durées et de rendre ainsi

${ }^{25}$ Les preuves textuelles ne manquent pas à l'appui de cette thèse. Trois occurrences suffiront ici. Page 82 de Durée et Simultanéité, on lit ceci: 'Est réel ce qui est mesuré par le physicien réel...'. Et page 207: 'Le Temps réel est [...] celui que le physicien perçoit et mesure...'. Bergson parle plus loin d'un temps 'mesuré directement, effectivement'. Page 209 enfin: 'Le Temps réel, marqué par l'horloge réelle...'. 
compte de leur écoulement simultané. Car il ne fait pas de doute que les jumeaux sont, en un certain sens, 'contemporains'. Et pour en rendre compte, il faut bien que les 'temps propres' écoulés ici et là soient en quelque manière commensurables. Telle est la situation théorique, envisagée par Bergson. La difficulté est que le 'temps propre', celui dont les équations du physicien garantissent l'invariance par changement de référentiel, est un temps auquel manque justement toute portée globale: c'est un temps local, privé de perspective; un temps-paramètre à certains égards moins artificiel que le temps-coordonné attaché aux systèmes de référence, mais qui n’a quand on y songe rien de spécifiquement temporel, si ce n'est par une espèce de 'transfusion de réalité' qui s'opère à chaque fois que la conscience se donne les moyens de 'parcourir' en pensée, en y apportant sa propre durée vécue, les lignes d'univers tracées dans l'espace-temps quadridimensionnel par les remous du monde. Nous voici donc avec des mesures locales, a priori disjointes, de durée matérielle, adossées à la basse continue que constitue la durée vécue des observateurs, durée caractérisée par un degré de tension générique, propre à l'espèce humaine. La tentative de retrouver sur une telle base l'unité proprement cosmologique de l'univers matériel est vouée à tourner court. C'est qu'elle accorde d'emblée trop d'importance à la durée psychologique, au risque de devoir très vite capituler devant les objections dirimantes des scientifiques Einstein au premier chef, dont on se souvient qu'il refusait tout bonnement d'admettre l'existence d'un temps du philosophe, un troisième temps qui ne serait ni le temps du physicien, ni le temps du psychologue... Mais surtout, il manque à cette base un concept clair de la perspective, qui rende compte de la possibilité d'étendre effectivement le temps sur l'espace à travers l'opération de la mesure. Il manque en somme une pensée de l'espace qui soit à la hauteur du problème.

Bergson aura au moins eu le mérite de mettre le doigt sur la difficulté principale, qui est de trouver un mode d'articulation cosmologique satisfaisant entre situation et perspective, déterminations locales et déterminations globales du temps. Sa confrontation avec la relativité einsteinienne lui aura permis de formuler une question capitale pour l'intelligence philosophique du temps physique: celle de la simultanéité ou de la coexistence des flux, coexistence irréductiblement temporelle et spatiale, que n'élimine nullement la mise en évidence du fait que la simultanéité instantanée (le 'maintenant' ponctuel) est relative au choix d'un référentiel (c'est-à-dire d'un temps-coordonnée étalé sur l'espace en totalité).

Il est vrai que la distinction traditionnelle entre durée et espace, multiplicité qualitative et multiplicité numérique, n'est pas ici d'un grand secours: au mieux, elle permet de nommer la difficulté. La question de la 
simultanéité devrait d'emblée se formuler en termes d'espace-temps. Mais il est tout aussi fâcheux de constater que les physiciens croient faire plaisir aux philosophes en leur accordant que la distinction entre durée vécue ('temps des consciences') et temps mesuré ('temps des horloges') conserve une validité et une pertinence. Nous proposons donc de bannir une fois pour toutes l'usage de ces expressions héritées d'un bergsonisme scolaire. On a vu en effet que toute l'affaire se jouait, dès le début, sur le terrain du temps mesuré: mettre à part le temps psychologique, comme s'il s'agissait d'un trésor à préserver des atteintes de l'activité spatialisante, n'est absolument d'aucune aide pour l'intelligence du problème. Langevin en son temps proposait de traduire 'durée vécue' par 'temps propre': cette concession aux philosophes allait dans le bon sens, mais elle ne faisait qu'aggraver les choses, puisqu'en l'absence d'une métrique temporelle unifiée sur l'espace-temps, les temps propres mesurés sur des trajectoires spatio-temporelles distinctes demeurent incommensurables, ce qui conforte l'idée d'une dispersion temporelle irréductible. C'est le cœur de l'affaire des jumeaux, séparés et pourtant contemporains. Einstein au moins avait eu la franchise de reconnaitre qu'il n'entendait rien au problème. Mais ce dernier fait retour aujourd'hui sous la forme d'une prolifération de définitions non-standard de la simultanéité. Si l'essentiel des débats philosophiques s'est finalement concentrée sur le caractère conventionnel ou non de telles définitions, il est intéressant de voir des métaphysiciens d'obédience analytique reprendre la question sous un angle non strictement épistémologique, en se penchant sur des modèles de coexistence régionale (selon un sens étendu, non ponctuel, de la localité) qui ont la caractéristique de pouvoir être directement extraits de la structure topologique de l'espace-temps de Minkowski.

\section{Entre fabulation et création: vivre et penser avec la science}

Revenons, en guise de conclusion, sur le rapport de la philosophie aux sciences. Bergson a posé les bases de sa méthode et engagé ses premières recherches dans un climat encore largement dominé par une foi positiviste dans les pouvoirs d'élucidation de l'intelligence mécaniste. Au tournant du siècle, il est sensible comme d'autres aux prémisses de ce qu'on allait bientôt appeler la 'crise des sciences', crise à laquelle son œuvre participe à sa façon en popularisant par exemple une lecture pragmatiste des théories scientifiques, envisagées comme outils intellectuels destinés à capturer les aspects mesurables de la réalité physique. Mais autant ne pas se le cacher: l'essentiel de ce que Bergson a pu écrire à ce sujet demeure en-deçà des révolutions qui ont durablement ébranlé l'image scientifique du monde dans le premier tiers du $\mathrm{XX}^{\mathrm{e}}$ siècle: nous pensons bien entendu à la relativité générale, dont Bergson ne dit quasiment rien, et plus encore à la mécanique quantique, à la biologie 
moléculaire ou aux théories de l'information, sans parler des refontes opérées sur le terrain logico-mathématiques. Or ces révolutions n'ont pas seulement conduit à une réforme profonde de certains schèmes catégoriels des sciences de la nature; elles ont aussi notablement transformé l'idée que l'on peut se faire de la signification et de la portée d'une théorie scientifique, en général. Leur diversité même complique singulièrement le projet de 'grande unification' censé accomplir l'idée de nature comme totalité soumise à des lois universelles. Il est crucial d'en tirer les conséquences pour que le bergsonisme élargi ne s'épuise pas à combattre des épouvantails - une science 'mécaniste' qui n'était déjà plus celle de nos grands-parents ${ }^{26}$.

On a évoqué plus haut la nécessité pour la métaphysique positive d'établir un rapport de 'distance critique' qui ne se ramène pas à l'élucidation des fondements épistémologiques ou métaphysiques des théories scientifiques. Or cela tient pour une part à la nature même de ces dernières, et l'on s'en rend mieux compte aujourd'hui. Pour le dire d'un mot: l'engagement ontologique d'une théorie scientifique est par nature limité. Elle ne dit le réel que d'une certaine manière, dont elle ne peut trouver en elle la pleine justification. Hume fut le premier à le dire à propos des sciences de la nature: une théorie scientifique échoue en permanence à trouver en elle son propre fondement. Ce constat, on pourrait l'étendre aujourd'hui aux sciences formelles, en tirant la leçon des théorèmes d'incomplétude énoncés par Gödel.

Il n'y a là rien d'étonnant dans la perspective bergsonienne: même une démonstration mathématique n'est finalement rien d'autre qu'un geste. Elle met toujours en œuvre, comme le montre Giuseppe Longo ${ }^{27}$, des principes de construction et pas seulement des principes de preuves. Cette déterritorialisation ne tient pas au contenu de la théorie, mais à son caractère structurellement pragmatique, ou pratique. Et nous retrouvons ici sous un autre angle le concept de redoublement d'immanence: une théorie scientifique nous parle, dans la pensée, d'un autre monde que celui de la pensée; elle s'ouvre aussi sur ce monde physique et biologique dans lequel nous sommes ancrés par les exigences de l'action. Ainsi, elle engage ce que l'on pourrait appeler une expérience élargie, ou encore une expérience décorrélée. En même temps que nous sommes dans le monde de nos pensées, nous sommes d'un monde physique

${ }^{26}$ À cet égard, Bachelard n'a évidemment pas tort de pointer l'obsession géométrique de Bergson, de lui reprocher son indifférence aux problématiques liées aux usages scientifiques des probabilités, ou plus généralement de l'algèbre. Cela ne l'empêche pas de célébrer en termes très bergsoniens ('élan spirituel', 'élan vital') les synthèses créatrices accomplies par la nouvelle mécanique (Le Nouvel esprit scientifique, Paris, Presses universitaires de France, 1934, p.183).

${ }^{27}$ Francis Bailly et Giuseppe Longo, Mathématiques et sciences de la nature: la Singularité physique du vivant, Paris, Hermann, 2006. 
qui n'est pas le nôtre. Les 'points de vues' de la science et de la conscience s'entrecroisent donc, mais ils ne sauraient se réduire l'un à l'autre. Bergson énonce ce principe à sa manière, en termes de 'systèmes d'images', dans un célèbre passage du début de Matière et Mémoire ${ }^{28}$. Voilà pourquoi sa démarche ne saurait se satisfaire ni de l'approche scientiste, ni de celle d'une phénoménologie idéaliste. Voilà aussi pourquoi on la retrouve au cœur des débats dans le paysage philosophique français contemporain, de Deleuze à Meillassoux, en passant par Pierre Montebello ou Renaud Barbaras.

Ajoutons que c'est également dans cette distance critique, impossible à combler, que vient se loger l'artiste tel que l'entend Bergson. Autrement dit, et en reprenant à notre compte l'heureuse formule de Jean-Marc Lévy-Leblond: la science n'est pas l'art. De même que la métaphysique positive ne saurait se réduire à l'engagement ontologique d'une théorie scientifique, le travail véritablement créateur de l'artiste ne consiste pas à simplement mettre en valeur, selon un régime métaphorique ou simplement illustratif, un concept ou une hypothèse que l'on trouverait tout faits dans la science. Les tableaux de Vinci ne sont pas conçus pour illustrer le nombre d'or, ni les installations de Duchamp pour figurer la quatrième dimension. C'est même tout le contraire. L'artiste est 'un distrait', écrit Bergson, c'est-à-dire qu'il est un voyant, non pas un savant. Il est là pour montrer ce que le savant lui-même ne peut pas voir, en raison du caractère limité de l'engagement ontologique de sa théorie. Et cette voyance ne nous voue pas, bien entendu, à un néo-romantisme un peu suspect. Elle peut prendre les formes les plus abstraites, les plus formelles en apparence: songeons au travail de François Morellet, chez qui l'opération artistique, sans cesser d'agir dans le sensible, devient parfois indiscernable du geste mathématique. Elle peut aussi conduire à envisager conjointement l'art et la science en régime expérimental: c'est le cas des fictions 'sous contrainte' inventées par Philippe Ramette, qui illustrent si bien l'affinité de certaines pratiques artistiques avec le travail rigoureux et risqué d'une science conçue comme 'cosa experimentale ${ }^{29}$ '.

Quoi qu'il en soit, il n'y a aucune convergence a priori entre création artistique et recherche technoscientifique. C'est plutôt au contraire sur leur complémentarité divergente qu'il faut s'appuyer, parce qu'elle donne toute sa valeur à l'exigence de fiction et d'interprétation propre à l'art, tout en laissant

\footnotetext{
28 'Aucune doctrine philosophique ne conteste que les mêmes images puissent entrer à la fois dans deux systèmes distincts, l'un qui appartient à la science, et où chaque image, n'étant rapportée qu'à ellemême, garde une valeur absolue, l'autre qui est le monde de la conscience, et où toutes les images se règlent sur une image centrale, notre corps, dont elles suivent les variations.' (Matière et Mémoire, op.cit., p.21).

${ }^{29}$ Nous reprenons cette expression, ainsi que les deux exemples, à Jean-Marc Lévy-Leblond.
} 
entrevoir une diversité d'articulations possibles avec la science. C'est en ce sens qu'il convient de réévaluer le rôle de la 'fonction fabulatrice' chez Bergson. On se souvient que cette dernière cimentait la morale et la religion closes. Mais on peut se demander si la dimension fabulatrice n'est pas toujours présente, non seulement dans la religion et la morale, mais dans la science même. Il y a à cela deux raisons principales.

D'abord, il n'est pas possible de séparer science et culture. Elles sont prises l'une dans l'autre. Et Lévy-Leblond a raison de faire remarquer à que ce n'est pas simplement dans la musique ou au théâtre, mais bien dans la science elle-même que l'on trouve, non seulement des chercheurs, mais des interprètes, qui peuvent être bons ou mauvais (auquel cas on leur réservera le titre de 'vulgarisateur'). Dans les sciences complexes d'aujourd'hui, la dimension d'interprétation est d'ailleurs de plus en plus clairement présente, ne serait-ce que par l'importance donnée à la notion de 'modèle' et au fait que les scientifiques sont systématiquement confrontés au problème de 'la sousdétermination de la théorie par l'expérience' (ce thème court dans la philosophie des sciences, de Duhem et Quine à Atlan).

Ensuite, comme le faisait remarquer Dominique Janicaud, il faut distinguer la science et sa puissance ${ }^{30}$. C'est entre les deux que nous trouvons la recherche technoscientifique, de moins en moins vouée à la science et de plus en plus vouée à sa puissance. Dit d'une autre manière, la recherche technoscientifique est aujourd'hui au cœur d'un dispositif techno-économique qui transforme la nature à un rythme et dans des proportions industrielles. La nature qui nous environne est aussi en notre pouvoir. Comme le montre Hans Jonas, ce qu'elle est dépend à présent de ce que l'homme peut et veut en faire. Elle ne relève donc plus simplement du fait mais aussi de la valeur, et le problème de la puissance de la science n'est pas qu'un problème théorique: c'est un problème axiologique qui nous place à l'articulation du savoir et de la croyance.

Mais fabulation rime avec clôture, et c'est de ce côté qu'il faut finalement tourner son attention si l'on veut donner toute sa portée à l'exigence critique qui soutient le rapport de la philosophie aux sciences. On peut en effet se demander s'il n'y a pas dans la relation entre théories scientifiques et préjugés scientistes une relation qui relève précisément de la clôture, une relation qui pousserait le monde scientifique à se refermer sur luimême, plutôt que de s'ouvrir aux univers culturels qui pourtant et paradoxalement le nourrissent et l'alimentent. Dans un autre sens, on peut aussi se demander ce qui distingue la fiction et la fabulation. Risquons ici une

${ }^{30}$ Dominique Janicaud, La Puissance du rationnel, Paris, Gallimard, 1985. 
hypothèse: derrière la fiction artistique et la fabulation, il n'y a pas le même régime de croyance. On peut s'en rendre compte en examinant la relation qui existe entre 'hallucination cognitive' dans la science et 'suspension d'incrédulité' dans la littérature, et peut-être plus particulièrement dans la littérature (ou le cinéma) de science-fiction. Le 'rayon N'est l'un des exemples classiques d'hallucination cognitive dans l'histoire des sciences. On pourrait également citer les controverses liées à la 'mémoire de l'eau'. Mais comment faire, ici encore, la part du bon grain et de l'ivraie? Comment démarquer la croyance rationnelle, fonction immanente à l'expérience théorique, et la simple fiction irréalisante ? Peut-on poser le problème autrement que dans les termes convenus d'une critique de l'idéologie spontanée des savants', ou alors d'une analyse psychologique de leurs biais motivationnels et cognitifs? Entre l'hypothèse du rayon $\mathrm{N}$ et celle de l'éther, plusieurs fois ressuscitée et abandonnée ces deux derniers siècles, où se situe la vraie distance? Et de même, entre la mémoire de l'eau et les épicycles de Ptolémée, ou encore les épi-gènes de la biologie moléculaire contemporaine ? Où passe la différence entre les 'sciences psychiques', auxquelles Bergson s'était sérieusement intéressé avant qu'elles ne rejoignent le registre infâmant de la parascience, et certaines recherches menées aujourd'hui aux frontières de l'art, de l'anthropologie et de la psychanalyse? Ces interrogations sont à l'horizon du bergsonisme élargi: elles dessinent en filigrane l'utopie concrète d'une science réellement ouverte.

\section{Envoi}

Faut-il le préciser? Sur toutes ces questions, et sur bien d'autres que nous n'avons même pas commencé à évoquer ici, l'idée n'est pas de chercher dans les sciences contemporaines - ou dans les formes ordinaires de leur pratique - des confirmations de telle ou telle prémonition bergsonienne. Qu'il s'agisse de la théorie de la perception en termes d'énaction, des bases neuropsychologiques de la mémoire ou de tout autre sujet de recherche, les intuitions ne sont pas faites pour être retrouvées et confirmées, mais bien plutôt compliquées à la lumière des questions qu'elles permettent de formuler. Bergson a mis au point une méthode de discernement et de création des problèmes; il en a posé pour son compte dont l'originalité continue de nous étonner. C'est à l'aune des questions qu'il nous lègue, et surtout de celles qu'il nous permet de poser à notre tour - non de tel ou tel point de doctrine, ni de 
sa vision du monde ${ }^{31}-$, qu'il convient de se demander aujourd'hui, collectivement, si nous voulons, si nous pouvons encore être bergsoniens.

Bref, nous possédons dès à présent un certain nombre de lignes de faits, qui ne vont pas aussi loin qu'il faudrait, mais que nous pouvons prolonger hypothétiquement. Je voudrais suivre avec vous quelques-unes d'entre elles. Chacune, prise à part, nous conduira à une conclusion simplement probable; mais toutes ensemble, par leur convergence, nous mettront en présence d'une telle accumulation de probabilités que nous nous sentirons, je l'espère, sur le chemin de la certitude. Nous nous en rapprocherons d'ailleurs indéfiniment, par le commun effort des bonnes volontés associées. Car la philosophie ne sera plus alors une construction, œuvre systématique d'un penseur unique. Elle comportera, elle appellera sans cesse des additions, des corrections, des retouches. Elle progressera comme la science positive. Elle se fera, elle aussi, en collaboration $^{32}$.

\section{Bibliographie}

BACHELARD, Gaston. Le Nouvel esprit scientifique. Paris: Presses universitaires de France, 1934.

BAILLY, Francis; LONGO, Giuseppe. Mathématiques et sciences de la nature: la Singularité physique du vivant. Paris: Hermann, 2006.

BERGSON, Henri. Matière et Mémoire [1896]. Paris: Presses universitaires de France, 2007.

. L'Évolution créatrice [1907]. Paris: PUF, 2007.

. Durée et Simultanéité [1922]. Paris: PUF, 2009.

. La Pensée et le mouvant [1934]. Paris: Presses universitaires de France,

2007.

. Écrits philosophiques. Paris: Presses universitaires de France, 2011.

BIANCO, Giuseppe. Après Bergson: portrait de groupe avec philosophe. Paris: Presses universitaires de France, 2015 (coll. Philosophie française contemporaine).

${ }^{31}$ Un monde qui dure? La belle affaire! La question est de savoir quelles raisons nous avons d'y tenir. Avis aux philosophes que séduiraient les passes d'armes entre 'présentistes' et 'éternalistes' (...). 32 Henri Bergson, L'Énergie spirituelle, Paris, Presses universitaires de France, 2009, p.4. 
DURING, Élie. "Bergson et la métaphysique relativiste". In: Annales Bergsoniennes, n.3, Paris: Presses universitaires de France, 2007 (coll. Épiméthée).

. "Temps kaléidoscopique et temps universel: la cosmologie bergsonienne à l'épreuve de la relativité". In: C. Riquier et F. Worms (dir.), Lire Bergson, Paris, Presses universitaires de France, 2011.

- "Vie et mort du cinématographe: de L'Évolution créatrice à Durée et simultanéite’. In: C. Riquier (dir.), Bergson, Paris, Cerf, 2012.

. ""Vous êtes subtil Monsieur Bergson", ou le bergsonisme peut-il s'enseigner ? ". In: A. Panero, S. Matton \&amp; M. Delbraccio (dir.). Bergson professeur, Louvain, Peteers, 2014.

GILSON, Étienne. "Souvenir de Bergson”. In: Revue de métaphysique et de morale, n.64, 1959.

JANICAUD, Dominique. La Puissance du rationnel. Paris: Gallimard, 1985.

LEVY-LEBLOND Jean-Marc. La Science n'est pas l'art. Paris: Hermann, 2010.

MIQUEL, Paul-Antoine. "Bergson et la science". In: P.-A. Miquel et F. Worms (dir.). Annales Bergsoniennes, n.3, Paris, Presses universitaires de France, 2007 (coll. Épiméthée).

. Bergson ou l'imagination métaphysique. Paris: Kimé, 2007.

. Le Vital: aspects physiques, aspects métaphysiques. Paris: Kimé, 2011.

. Bergson dans le miroir des sciences. Paris: Kimé, 2014.

. Sur le concept de nature. Paris: Hermann, 2015.

MONTEBELLO, Pierre. L'autre métaphysique: essai sur la philosophie de la nature, Ravaisson, Tarde, Nietzsche et Bergson. Paris: Desclée de Brouwer, 2003.

RANCIERE Jacques. La Chair des mots. Paris: Galilée, 1999.

RIQUIER Camille. "Voir et cependant ne pas croire" Intuition et méthode chez Bergson”. In: Transparaître, n.1, 'l'intuition', Décembre, 2007. . Archéologie de Bergson. Paris: Presses universitaires de France, 2009.

RUYER, Raymond. Néo-finalisme [1952]. Rééd. Paris: PUF, 2012.

SIMONDON, Gilbert. L'Individuation à la lumière des notions de forme et d'information. Grenoble: Jérôme Millon, 2005. 
WORMS, Frédéric. Bergson ou les deux sens de la vie. Paris:Presses universitaires de France, 2004.

. La Vie qui unit et qui sépare. Paris: Payot, 2013. 